\title{
When natural selection should optimize speed-accuracy trade-offs
}

\author{
Angelo Pirrone ${ }^{1,2}$, Tom Stafford ${ }^{1}$ and James A. R. Marshall ${ }^{2,3 *}$ \\ ${ }^{1}$ Department of Psychology, University of Sheffield, Sheffield, UK \\ ${ }^{2}$ Kroto Research Institute, University of Sheffield, Sheffield, UK \\ ${ }^{3}$ Department of Computer Science, University of Sheffield, Sheffield, UK \\ *Correspondence: james.marshall@sheffield.ac.uk
}

Edited by:

Dominic Standage, Queen's University, Canada

Reviewed by:

Joshua I. Gold, University of Pennsylvania, USA

Kenway Louie, New York University, USA

Keywords: decision-making, value, reward, error, Bayes risk, drift-diffusion, mechanism, evolution

\section{INTRODUCTION}

In psychology and neuroscience, and in other disciplines studying decisionmaking mechanisms, it is often assumed that optimal decision-making means statistical optimality. This is attractive because statistically optimal decision procedures are known, can be simply implemented in biologically-plausible models, and because such models have been shown to give good fits to behavioural as well as neural data. Here we question when statistical optimality is the kind of optimality we should expect natural selection to aim towards, by considering what kinds of loss function should be optimised under different behavioural scenarios. In laboratory settings subjects are often rewarded only on making a correct choice, so optimisation of a zero-one loss function is appropriate, and this is achieved by implementing a statisticallyoptimal decision procedure that gives the best compromise between speed and accuracy of decision-making. Many naturalistic decisions may also be described by such a loss function; however others, such as selecting food items of potentially different value, appear to be different since the animal is rewarded by the value of the item it chooses regardless of whether it was the best available. We argue that most naturalistic decisions are value-based. Mechanisms that optimise speed-accuracy trade-offs need to be parameterised, using information about the decision problem, in order to deal with value-based decisionmaking. Mechanisms for value-sensitive decision-making have been described, however, which adaptively change between decision-making strategies without the need for continual re-parameterisation.

\section{SPEED-ACCURACY TRADE-OFFS}

It is usually assumed that decision-makers have to decide to be either fast or accurate. When speed is important mistakes are more frequent, while when accuracy is needed decisions are slower. This obvious problem is defined as the speedaccuracy trade-off and is a distinctive feature of many types of decision making (Wickelgren, 1977).

The speed-accuracy trade-off can be explained within the theoretical framework of sequential sampling models of decision making that have been shown to fit behavioral and neural data from human and animal choice tasks (Ratcliff and Rouder, 2000; Ratcliff et al., 2003, 2004; Ratcliff and Smith, 2004; Busemeyer et al., 2013). In particular, the Drift Diffusion Model (DDM; Ratcliff, 1978) describes choice between two alternatives (see Smith and Ratcliff, 2004; Bogacz et al., 2006; Basten et al., 2010) and recently has been shown also to be quantitatively accurate in describing trinary choices (Krajbich and Rangel, 2011) and value-based choices (Krajbich et al., 2010; Milosavljevic et al., 2010; Krajbich and Rangel, 2011; Krajbich et al., 2012), suggesting that the DDM can be thought of as a unifying computational framework for describing decision making (Basten et al., 2010). Moreover, Bogacz et al. (2006) have demonstrated that several connectionist decision-making models can approximate the DDM under specific conditions. The DDM is a special case of the statisticallyoptimal Sequential Probability Ratio Test (SPRT; Wald, 1947; Wald and Wolfowitz, 1948). In the DDM noisy sensory evidence supporting the alternatives is integrated over time until the net evidence in favor of one alternative exceeds a certain positive or negative threshold value, precipitating a decision for the corresponding alternative. These thresholds can be varied to compromise optimally between the average speed and accuracy of decisions.

\section{SPEED-VALUE TRADE-OFFS}

In situations where decisions are rewarded according to whether they are correct or not, optimizing the speed-accuracy trade-off is sensible. When decisions are rewarded according to the value of the option chosen, however, a different criterion needs to be optimized. This can be illustrated with the simplest case of choosing between two equal value options; here there is no decision accuracy, since choosing either option is "correct." Similarly, there is no difference in average evidence for which of the two options is more valuable, meaning that the SPRT/DDM will only reach a decision by integrating sufficient noise to cross a decision threshold. Thus in this scenario there is no speedaccuracy trade-off to manage; the optimal decision is to choose anything as quickly as possible. The fundamental insight is that for certain decisions, speed-value tradeoffs are more appropriate to optimize, rather than speed-accuracy trade-offs. 
The SPRT/DDM can be optimized to take account of the value of the alternatives but, as we discuss here, doing so requires knowledge of the decision problem faced. The thresholds for an optimal decision depend on the goals of the decision maker and are task specific. By way of example, one route to accounting for the values associated with different decision outcomes is to minimize an extended version of the Bayes Risk (BR). BR is a linear combination of expected decision delay and expected terminal decision loss, first proposed by Wald and Wolfowitz (1948), and assumes that decision makers seek to minimize a cost function that is the weighted sum of decision times (DTs) and error rate (ERs). This was subsequently extended by Edwards to also account for non-zero rewards for incorrect decisions (Edwards, 1965; Bogacz et al., 2006). Formally Edwards' extension of BR, which implements Wald and Wolfowitz's version as a special case, can be defined as

$$
B R_{E}=c_{1} D T+c_{2}\left(\begin{array}{c}
E R \\
1-E R
\end{array}\right)
$$

where $c_{1}$ is the cost of observing the stimulus per unit time, while $c_{2}$ is a row-vector specifying the payoffs from incorrect and correct choices (Bogacz et al., 2006). If $c_{2}=\left(\begin{array}{l}k 0\end{array}\right)$, where $k>0$ is a constant, then Wald and Wolfowitz's original BR is recovered. Several studies demonstrate that, under specific circumstances, subjects choose decision thresholds close to those that minimize $B R_{E}$ (Busemeyer and Rapoport, 1988; Mozer et al., 2002). Bayes risk is not the only criterion proposed to date that decision-makers might optimize. Bogacz et al. survey alternatives, such as reward-rate, however, these alternatives are all calculated based on decision-accuracy, which requires explicit parameterizations based on the values of correct and incorrect choices (Bogacz et al., 2006). We therefore concentrate our analysis on Bayes risk. Bayes risk can be used to optimize value-sensitive decisionmaking; for example in a decision between two equal alternatives, each having value $v$ if chosen, we would set the vector $c_{2}=$ $(v v)$ (e.g., dashed green line in Figure 1), thus simplifying Equation (1) above to

$$
B R_{E}=c_{1} D T+v .
$$

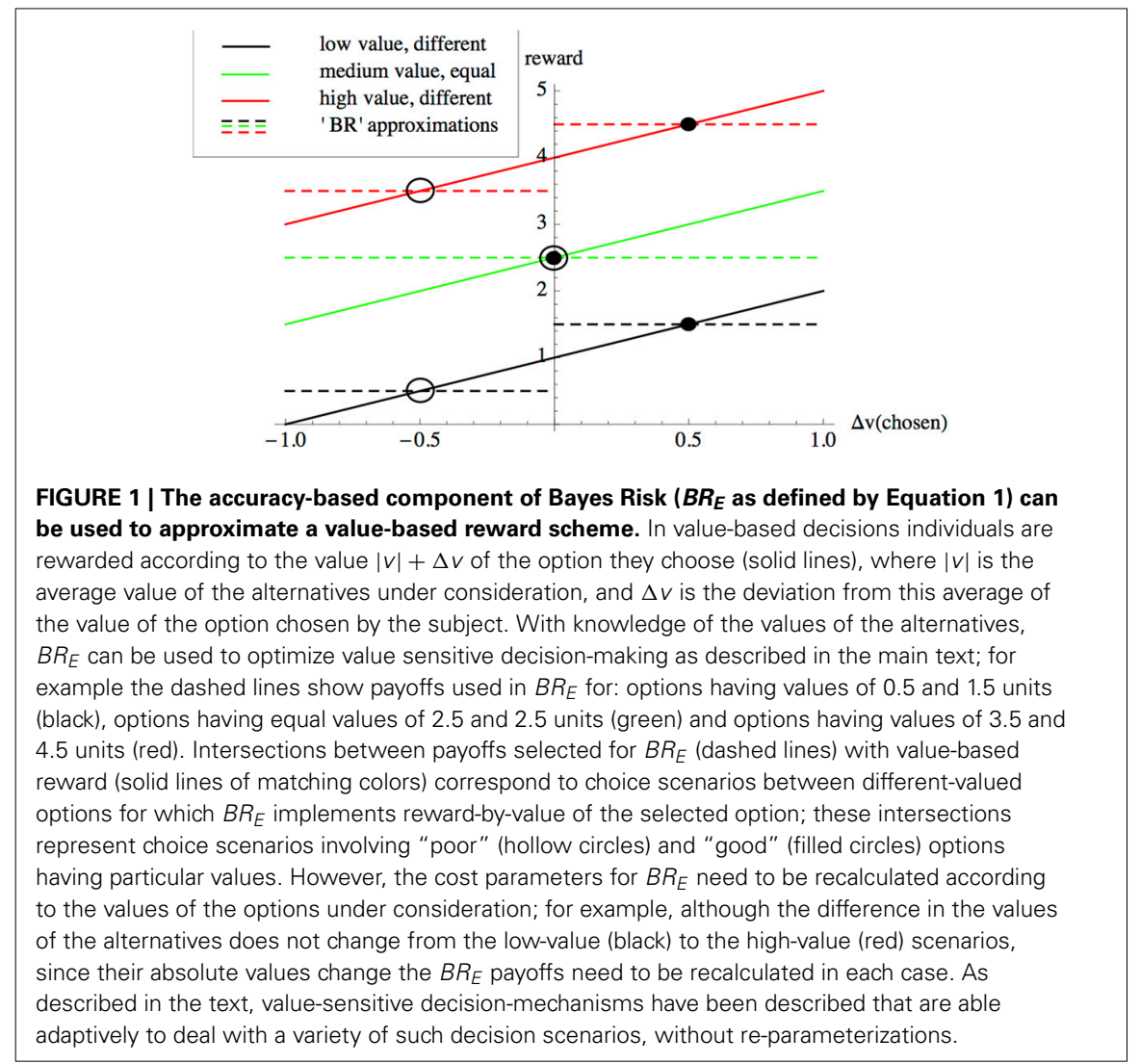

Equation (2) shows us that, intuitively, an optimal decision-maker in our equalalternatives scenario should minimize decision-time $D T$, since doing so incurs no penalty as the error rate ER no longer features. However, using Bayes risk in this way requires the values of the alternatives to be known on a case by case basis, as shown in Figure 1. Subjects might learn the values of incorrect and correct choices over time, for example when trials are blocked in psychophysical experiments (see Bogacz et al., 2006). However, in the following we argue that in most naturalistic decision scenarios decisionmakers will not have this opportunity, and will therefore use other mechanisms that directly optimize speed-value tradeoffs, rather than optimizing decisions indirectly via optimization of the speedaccuracy trade-off with an appropriate payoff vector $c_{2}$.

\section{NATURALISTIC DECISIONS ARE USUALLY VALUE-BASED}

We argue that most naturalistic decisions faced by animals, including humans, are value-based, in that the animal is rewarded according to the value of the option it chooses. Such a view on decision-making is not new to behavioral ecologists, where a long tradition exists of studying behaviors such as mate choice and foraging (Davies et al., 2012) or nest-site selection (Stroeymeyt et al., 2014). Recently many studies have focused on how value and reward are represented and integrated during the decision process (Platt and Glimcher, 1999; Sugrue et al., 2004; Padoa-Schioppa and Assad, 2006; Rangel et al., 2008; Kable and Glimcher, 2009; Krajbich et al., 2010; Philiastides et al., 2010; Hare et al., 2011; Krajbich and Rangel, 2011; Louie and Glimcher, 2012; Tsetsos et al., 2012; Cassey et al., 2013; Towal et al., 2013); however, in psychology and neuroscience, experiments are usually designed such that there is always a correct choice, and only correct choices are rewarded (see Gold and Shadlen, 2003; Bogacz et al., 2006). While studying behavior in psychophysical tasks is beneficial in that it gives a well-controlled decision environment, our point is that only rewarding subjects when they make correct choices may not correspond to the 
kind of decisions animals, and their neural circuitry, have typically evolved to deal with. Even in the value-based decision experiments cited above, which are analyzed using the DDM, it is typical to only present subjects with a choice between options known to have different values. Moreover, even though some studies have looked at how reward information is integrated (Rorie et al., 2010; Gao et al., 2011), much of this work has not yet focused on the tradeoff between value and speed. While usually in the decision-making literature the optimal behavior is to optimize speed-accuracy trade-offs, and subjects can apparently do this (Busemeyer and Rapoport, 1988; Bogacz et al., 2006), we argue that these scenarios are not representative of many naturalistic settings, and that there is great value in considering how subjects make value-sensitive decisions and how these should be optimized. In the following section we discuss theory that may be useful for this.

At least one important class of naturalistic decisions does require optimization of speed-accuracy trade-offs; these are lifeor-death decisions. If we analyze for example the case of an animal attempting to forage while avoiding predators (Trimmer et al., 2008), a slow-but-accurate decision would mean being killed by the predator, a maximal loss. On the other hand if the decision is fast-but-inaccurate the animal would escape even when the stimulus is not a predator, and this would mean losing food. The best strategy for the animal is thus that which optimizes the speed-accuracy trade-off, taking into account the payoffs arising from the different decision outcomes; hence Trimmer et al.'s hypothetical animal is modeled with a single-threshold DDM, with evidence sufficient to cross that single decision threshold leading to the animal taking anti-predator action such as running away.

\section{MECHANISMS FOR VALUE- SENSITIVE DECISION-MAKING}

Recent modeling work inspired by studying another value-sensitive decisionmaking system, collective nest-site selection by honeybees (Seeley et al., 2012), has described a very simple mechanism able to adaptively account for the value of different decision outcomes, with minimal parameter tuning (Pais et al.,
2013). This simple model implements a variety of sophisticated decision-making strategies; for example, when equal but low-value alternatives are presented, a decision deadlock is maintained that can be broken should a third, higher-value alternative, be made available. However, if equal-but-high-value alternatives are presented, or sufficient time passes, deadlock is spontaneously and randomly broken (Pais et al., 2013). This is particularly interesting, since the classic DDM is insensitive to the absolute value of the alternatives under consideration, and only integrates the difference in their values. When differences between alternative values are sufficient, the value-sensitive mechanism of Pais et al. becomes closer to a classic DDM, allowing speed-accuracy trade-offs to be managed, although not optimized, through modification of decision thresholds. All of the different behavioral regimes of the model arise without direct parameterizations regarding alternatives' values, simply through the dependence of the model's dynamics on the mean values of inputs to its integrator populations; this allows the model to adaptively respond to different decision scenarios on a trial-by-trial basis, which cannot be achieved in pure DDM models without the decision-maker having access to explicit information on the decision-task at hand. Modifications to DDM-type models have been proposed to deal with trial-by-trial variability such as online estimation of task parameters (Deneve, 2012) or the use of time-dependent change in parameters such as decision-thresholds, urgency signals or asymmetry of inhibition (Ditterich, 2006; Hanks et al., 2011; Drugowitsch et al., 2012; Thura et al., 2012); fundamentally, however, these modifications are still interpreted under the assumption that decision speed vs accuracy is the tradeoff to be maximized, unlike the model of Pais et al. (2013) in which the dynamics are naturally interpreted in terms of value vs time trade-offs. Pais et al.'s mechanism also exhibits other characteristics of natural value-discrimination systems, such as Weber's law of just-noticeable difference; interestingly Weber's law arises from the deterministic dynamics of the mechanism rather than from noise processes (Pais et al., 2013) (cf. Deco and Rolls, 2006; Deco et al., 2007). Finally, it is important to note that the DDM cannot account for the non-linearity that characterizes many decision making dynamics (e.g., food recruitment by social insects; Nicolis and Deneubourg, 1999) while the model of Pais et al. (2013) is non-linear.

\section{CONCLUSION}

The study of speed-accuracy trade-offs has been tremendously fruitful for psychology, neuroscience and animal behavior, and will doubtless prove fruitful for many years to come. Yet as we have argued here most naturalistic decisions, which animals' brains should have evolved to optimize, are value-based rather than accuracybased. This leads us to argue that the drift-diffusion model, which optimizes speed-accuracy trade-offs, is not an ideal computational framework to describe value-based decision-making; although it has had some success in describing particular experiments on value-based decision-making, discussed in the section "Speed-Accuracy Trade-Offs," as we have shown here the DDM requires special case-by-case parameterizations to implement true value-based decision-making. We suggest that this limits the generality of the DDM as a unifying framework for all ecologically-relevant decision-making problems. However, recent theory has presented mechanisms that can manage value-sensitive decision problems without the additional informational requirements of the DDM. At the same time, experimental and theoretical psychologists and neuroscientists have started to tackle problems of value-based decision-making. We have presented our arguments for value in terms of animal decision-making, but unicellular organisms and individual cells also make decisions (e.g., Perkins and Swain, 2009; Latty and Beekman, 2011), and value is likely to be similarly important for these. We believe that the evolutionary perspective we have presented here should motivate further research into value-sensitivity and decision-making.

\section{AUTHOR CONTRIBUTIONS}

James A. R. Marshall conceived of the paper; James A. R. Marshall, Angelo Pirrone, and Tom Stafford discussed the material; James A. R. Marshall developed 
the formal argument; Angelo Pirrone and James A. R. Marshall drafted the paper and all authors approved its content.

\section{FUNDING}

Angelo Pirrone is supported by the University of Sheffield Studentship Network in Neuroeconomics.

\section{ACKNOWLEDGMENTS}

We thank Jochen Ditterich, Ian Krajbich, and Konstantinos Tsetsos for helpful comments on the manuscript.

\section{REFERENCES}

Basten, U., Biele, G., Heekeren, H. R., and Fiebach, C. J. (2010). How the brain integrates costs and benefits during decision making. Proc. Natl. Acad. Sci. U.S.A. 107, 21767-21772. doi: 10.1073/pnas.0908104107

Bogacz, R., Brown, E., Moehlis, J., Holmes, P., and Cohen, J. D. (2006). The physics of optimal decision making: a formal analysis of models of performance in two-alternative forced-choice tasks. Psychol. Rev. 113:700. doi: 10.1037/0033295X.113.4.700

Busemeyer, J., Diederich, A., Heathcote, A., Rangel, A., Rieskamp, J., and Usher, M. (2013). "Sequential sampling models representing a unifying framework of human decision making," in Symopsium, COGSCI Annual Meeting (Berlin).

Busemeyer, J. R., and Rapoport, A. (1988). Psychological models of deferred decision making. J. Math. Psychol. 32, 91-134. doi: 10.1016/0022-2496(88)90042-9

Cassey, T. C., Evens, D. R., Bogacz, R., Marshall, J. A. R., and Ludwig, C. J. H. (2013). Adaptive sampling of information in perceptual decisionmaking. PLoS ONE 8:e78993. doi: 10.1371/journal.pone. 0078993

Davies, N. B., Krebs, J. R., and West, S. A. (2012). An Introduction to Behavioural Ecology, 4th Edn. Hoboken, NJ: John Wiley \& Sons.

Deco, G., and Rolls, E. T. (2006). Decision-making and weber's law: a neurophysiological model. Eur. J. Neurosci. 24, 901-916. doi: 10.1111/j.14609568.2006.04940.x

Deco, G., Scarano, L., and Soto-Faraco, S. (2007). Weber's law in decision making: integrating behavioral data in humans with a neurophysiological model. J. Neurosci. 27, 11192-11200. doi: 10.1523/JNEUROSCI.1072-07.2007

Deneve, S. (2012). Making decisions with unknown sensory reliability. Front. Neurosci. 6:75. doi: 10.3389/fnins.2012.00075

Ditterich, J. (2006). Stochastic models of decisions about motion direction: behavior and physiology. Neural Netw. 19, 981-1012. doi: 10.1016/j.neunet. 2006.05.042

Drugowitsch, J., Moreno-Bote, R., Churchland, A. K., Shadlen, M. N., and Pouget, A. (2012). The cost of accumulating evidence in perceptual decision making. J. Neurosci. 32, 3612-3628. doi: 10.1523/JNEUROSCI.4010-11.2012

Edwards, W. (1965). Optimal strategies for seeking information: models for statistics, choice reaction times, and human information processing. $J$. Math. Psychol. 2, 312-329. doi: 10.1016/00222496(65)90007-6

Gao, J., Tortell, R., and McClelland, J. L. (2011). Dynamic integration of reward and stimulus information in perceptual decision-making. PLoS ONE 6:e16749. doi: 10.1371/journal.pone. 0016749

Gold, J. I., and Shadlen, M. N. (2003). The influence of behavioral context on the representation of a perceptual decision in developing oculomotor commands. J. Neurosci. 23, 632-651. doi: 10.1146/annurev.neuro.29.051605.113038

Hanks, T. D., Mazurek, M. E., Kiani, R., Hopp, E., and Shadlen, M. N. (2011). Elapsed decision time affects the weighting of prior probability in a perceptual decision task. J. Neurosci. 31, 6339-6352. doi: 10.1523/JNEUROSCI.561310.2011

Hare, T. A., Schultz, W., Camerer, C. F., O’Doherty, J. P., and Rangel, A. (2011). Transformation of stimulus value signals into motor commands during simple choice. Proc. Natl. Acad. Sci. U.S.A. 108, 18120-18125. doi: 10.1073/pnas.1109322108

Kable, J. W., and Glimcher, P. W. (2009). The neurobiology of decision: consensus and controversy. Neuron 63, 733-745. doi: 10.1016/j.neuron.2009. 09.003

Krajbich, I., Armel, C., and Rangel, A. (2010). Visual fixations and the computation and comparison of value in simple choice. Nat. Neurosci. 13, 1292-1298. doi: 10.1038/nn.2635

Krajbich, I., Lu, D., Camerer, C., and Rangel, A. (2012). The attentional drift-diffusion model extends to simple purchasing decisions. Front. Psychol. 3:193. doi: 10.3389/fpsyg.2012. 00193

Krajbich, I., and Rangel, A. (2011). Multialternative drift-diffusion model predicts the relationship between visual fixations and choice in valuebased decisions. Proc. Natl. Acad. Sci. U.S.A. 108, 13852-13857. doi: 10.1073/pnas. 1101328108

Latty, T., and Beekman, M. (2011). Speed-accuracy trade-offs during foraging decisions in the acellular slime mould physarum polycephalum. Proc. $R$. Soc. B Biol. Sci. 278, 539-545. doi: 10.1098/rspb. 2010.1624

Louie, K., and Glimcher, P. W. (2012). Efficient coding and the neural representation of value. Ann. N.Y. Acad. Sci. 1251, 13-32. doi: 10.1111/j.17496632.2012.06496.x

Milosavljevic, M., Malmaud, J., Huth, A., Koch, C., and Rangel, A. (2010). The drift diffusion model can account for value-based choice response times under high and low time pressure. Judge. Decis. Making 5, 437-449. doi: 10.2139/ssrn.1901533

Mozer, M. C., Colagrosso, M. D., and Huber, D. E. (2002). "A rational analysis of cognitive control in a speeded discrimination task," in Advances in Neural Information Processing Systems, Vol. 11, eds T. Dietterich, S. Becker, and Z. Ghahramani (Cambridge, MA: MIT Press), 51-57.

Nicolis, S. C., and Deneubourg, J.-L. (1999). Emerging patterns and food recruitment in ants: an analytical study. J. Theor. Biol. 198, 575-592. doi: 10.1006/jtbi.1999.0934

Padoa-Schioppa, C., and Assad, J. A. (2006). Neurons in the orbitofrontal cortex encode economic value. Nature 441, 223-226. doi: 10.1038/nature04676
Pais, D., Hogan, P. M., Schlegel, T., Franks, N. R., Leonard, N. E., and Marshall, J. A. R. (2013). A mechanism for value-sensitive decision-making. PLoS ONE 8:e73216. doi: 10.1371/journal.pone.0073216

Perkins, T. J., and Swain, P. S. (2009). Strategies for cellular decision-making. Mol. Syst. Biol. 5, 1-15. doi: 10.1038/msb.2009.83

Philiastides, M. G., Biele, G., and Heekeren, H. R. (2010). A mechanistic account of value computation in the human brain. Proc. Natl. Acad. Sci. U.S.A. 107, 9430-9435. doi: 10.1073/pnas. 1001732107

Platt, M. L., and Glimcher, P. W. (1999). Neural correlates of decision variables in parietal cortex. Nature 400, 233-238. doi: 10.1038/22268

Rangel, A., Camerer, C., and Montague, P. R. (2008). A framework for studying the neurobiology of value-based decision making. Nat. Rev. Neurosci. 9 , 545-556. doi: 10.1038/nrn2357

Ratcliff, R. (1978). A theory of memory retrieval. Psychol. Rev. 85:59. doi: 10.1037/0033-295X. 85.2.59

Ratcliff, R., Gomez, P., and McKoon, G. (2004). A diffusion model account of the lexical decision task. Psychol. Rev. 111:159. doi: 10.1037/0033295X.111.1.159

Ratcliff, R., and Rouder, J. N. (2000). A diffusion model account of masking in two-choice letter identification. J. Exp. Psychol. 26:127. doi: 10.1037/0096-1523.26.1.127

Ratcliff, R., and Smith, P. L. (2004). A comparison of sequential sampling models for two-choice reaction time. Psychol. Rev. 111:333. doi: 10.1037/0033295X.111.2.333

Ratcliff, R., Thapar, A., and Mckoon, G. (2003). A diffusion model analysis of the effects of aging on brightness discrimination. Percept. Psychophys. 65, 523-535. doi: 10.3758/BF03194580

Rorie, A. E., Gao, J., McClelland, J. L., and Newsome, W. T. (2010). Integration of sensory and reward information during perceptual decision-making in lateral intraparietal cortex (lip) of the macaque monkey. PLoS ONE 5:e9308. doi: 10.1371/journal.pone. 0009308

Seeley, T. D., Visscher, P. K., Schlegel, T., Hogan, P. M., Franks, N. R., and Marshall, J. A. R. (2012), Stop signals provide cross inhibition in collective decision-making by honeybee swarms. Science 335 , 108-111. doi: 10.1126/science. 1210361

Smith, P. L., and Ratcliff, R. (2004). Psychology and neurobiology of simple decisions. Trends Neurosci. 27, 161-168. doi: 10.1016/j.tins.2004.01.006

Stroeymeyt, N., Jordan, C., Mayer, G., Hovsepian, S., Giurfa, M., and Franks, N. (2014). Seasonality in communication and collective decision-making in ants. Proc. R. Soc. B. Biol. Sci. 281:20133108. doi: $10.1098 / \mathrm{rspb} .2013 .3108$

Sugrue, L. P., Corrado, G. S., and Newsome, W. T. (2004). Matching behavior and the representation of value in the parietal cortex. Science 304, 1782-1787. doi: 10.1126/science. 1094765

Thura, D., Beauregard-Racine, J., Fradet, C.-W., and Cisek, P. (2012). Decision making by urgency gating: theory and experimental support. J. Neurophysiol. 108, 2912-2930. doi: 10.1152/jn.01071.2011

Towal, R. B., Mormann, M., and Koch, C. (2013). Simultaneous modeling of visual saliency and 
value computation improves predictions of economic choice. Proc. Natl. Acad. Sci. U.S.A. 110, E3858-E3867. doi: 10.1073/pnas.1304429110

Trimmer, P. C., Houston, A. I., Marshall, J. A. R., Bogacz, R., Paul, E. S., Mendl, M. T., et al. (2008). Mammalian choices: combining fast-butinaccurate and slow-but-accurate decision-making systems. Proc. R. Soc. B Biol. Sci. 275, 2353-2361. doi: 10.1098/rspb.2008.0417

Tsetsos, K., Chater, N., and Usher, M. (2012). Salience driven value integration explains decision biases and preference reversal. Proc. Natl. Acad. Sci. U.S.A. 109, 9659-9664. doi: 10.1073/pnas.1119 569109

Wald, A. (1947). Sequential Analysis. New York, NY: Wiley and Sons.
Wald, A., and Wolfowitz, J. (1948). Optimum character of the sequential probability ratio test. Ann. Math. Stat. 19, 326-339. doi: 10.1214/aoms/1177 730197

Wickelgren, W. A. (1977). Speed-accuracy tradeoff and information processing dynamics. Acta Psychol. 41, 67-85. doi: 10.1016/0001-6918(77) 90012-9

Conflict of Interest Statement: The authors declare that the research was conducted in the absence of any commercial or financial relationships that could be construed as a potential conflict of interest.

Received: 21 January 2014; accepted: 26 March 2014; published online: 10 April 2014.
Citation: Pirrone A, Stafford $T$ and Marshall JAR (2014) When natural selection should optimize speed-accuracy trade-offs. Front. Neurosci. 08:73. doi: 10.3389/fnins.2014.00073

This article was submitted to Decision Neuroscience, a section of the journal Frontiers in Neuroscience.

Copyright (c) 2014 Pirrone, Stafford and Marshall. This is an open-access article distributed under the terms of the Creative Commons Attribution License (CC BY). The use, distribution or reproduction in other forums is permitted, provided the original author(s) or licensor are credited and that the original publication in this journal is cited, in accordance with accepted academic practice. No use, distribution or reproduction is permitted which does not comply with these terms. 